Supporting Information

\title{
Evidence of Molecular Structure Dependent Charge Transfer Between Isoindigo-Based Polymers and Fullerene
}

Tzung-Han Lai ${ }^{1}$, Iordania Constantinou ${ }^{1}$, Caroline M. Grand ${ }^{2}$, Erik D. Klump ${ }^{1}$, Sujin Baek ${ }^{1}$, Hsien Yi Hsu ${ }^{3}$, Sai-Wing Tsang ${ }^{4}$, Kirk S. Schanze ${ }^{3}$, John R. Reynolds ${ }^{2}$ and Franky So ${ }^{1,5^{*}}{ }^{2}$

${ }^{1}$ Department of Materials Science and Engineering, University of Florida, Gainesville, FL, US

${ }^{2}$ School of Chemistry and Biochemistry, School of Materials Science and Engineering, Center for Organic Photonics and Electronics, Georgia Tech Polymer Network, Georgia Institute of Technology, Atlanta, GA, USA

${ }^{3}$ Department of Chemistry, University of Florida, Gainesville, FL, USA

${ }^{4}$ Department of Physics and Materials Science, City University of Hong Kong, Hong Kong, China

${ }^{5}$ Department of Materials Science and Engineering, North Carolina State University, Raleigh, NC, USA.

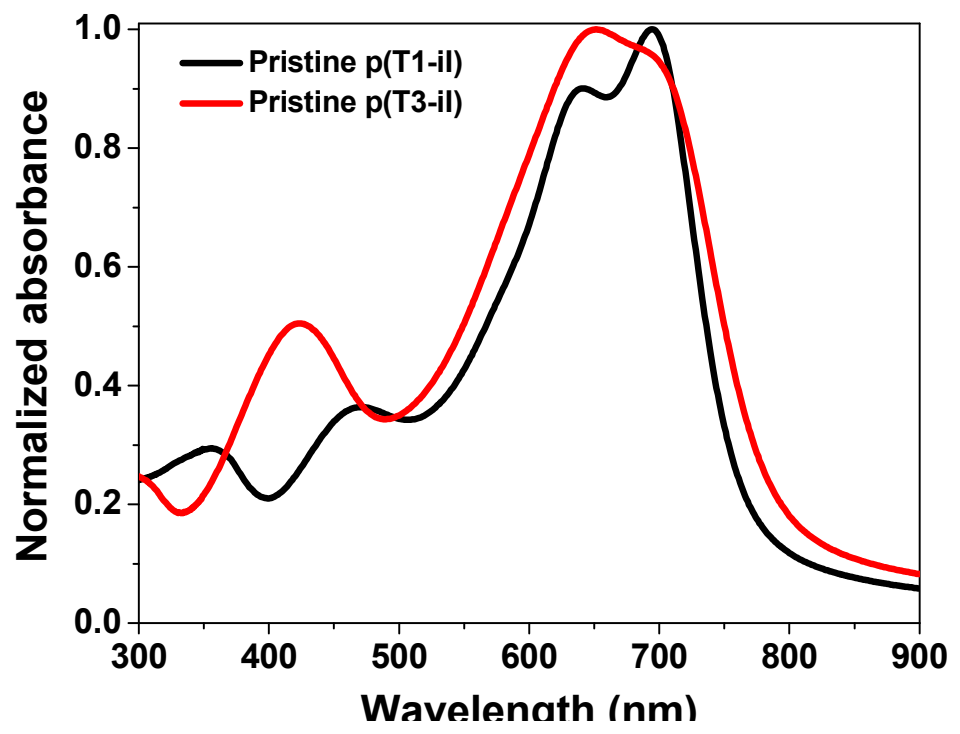

Figure S1. Normalized absorption spectra for P(T1-iI) and P(T3-iI) polymers. 


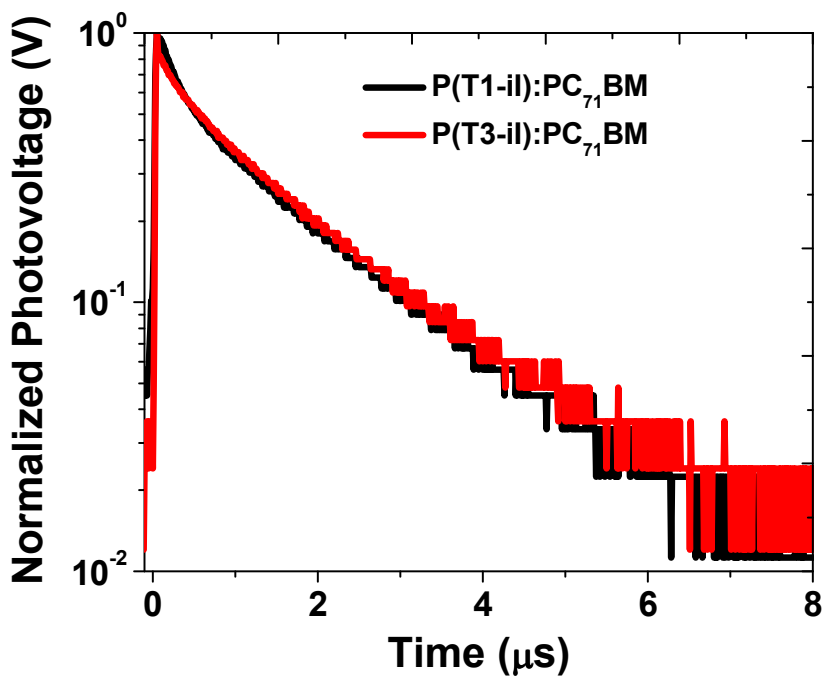

Figure S2. Transient photovoltage (TPV) decay measured for P(T1-iI):PC71BM and P(T3-iI):PC71BM devices. The data show a carrier lifetime of $1.38 \mu$ s and $1.25 \mu$ s respectively. This indicates nearly identical bimolecular recombination rates.

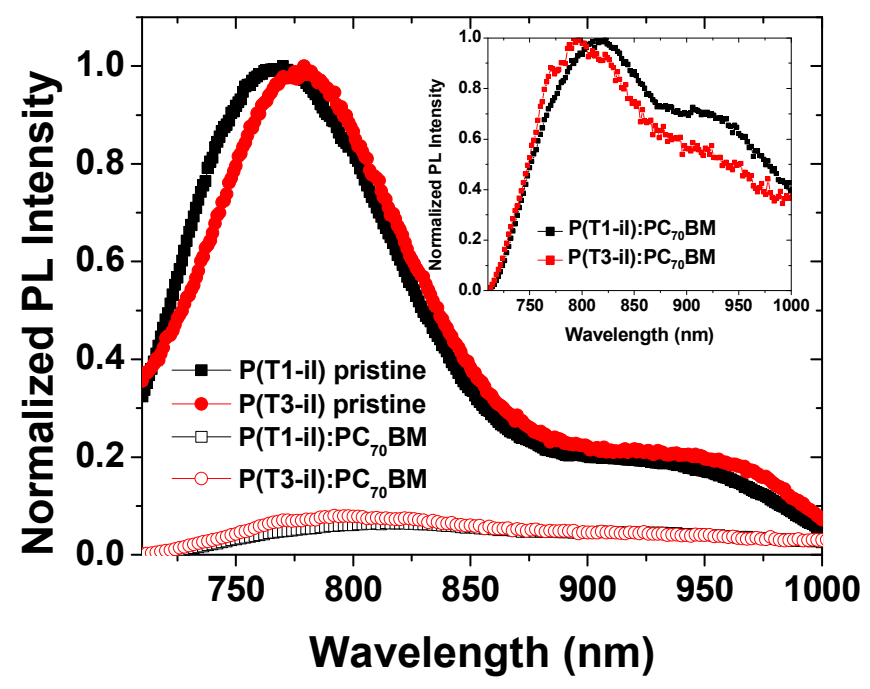

Figure S3. Normalized steady-state photoluminescence spectra for pristine $\mathrm{P}(\mathrm{T} 1-\mathrm{iI})$ and $\mathrm{P}(\mathrm{T} 3-\mathrm{iI})$ films along with the PL spectra for the $\mathrm{P}(\mathrm{T} 1-\mathrm{iI}): \mathrm{PC}_{70} \mathrm{BM}$ and $\mathrm{P}(\mathrm{T} 3-\mathrm{iI}): \mathrm{PC}_{70} \mathrm{BM}$ blends relative to the corresponding pristine polymers. $\mathrm{PL}$ is almost completely quenched after blending with $\mathrm{PC}_{70} \mathrm{BM}$ in both cases. Inset: Normalized PL spectra for $\mathrm{P}(\mathrm{T} 1-\mathrm{iI}): \mathrm{PC}{ }_{70} \mathrm{BM}$ and $\mathrm{P}(\mathrm{T} 3-\mathrm{iI}): \mathrm{PC}{ }_{70} \mathrm{BM}$ blends. 

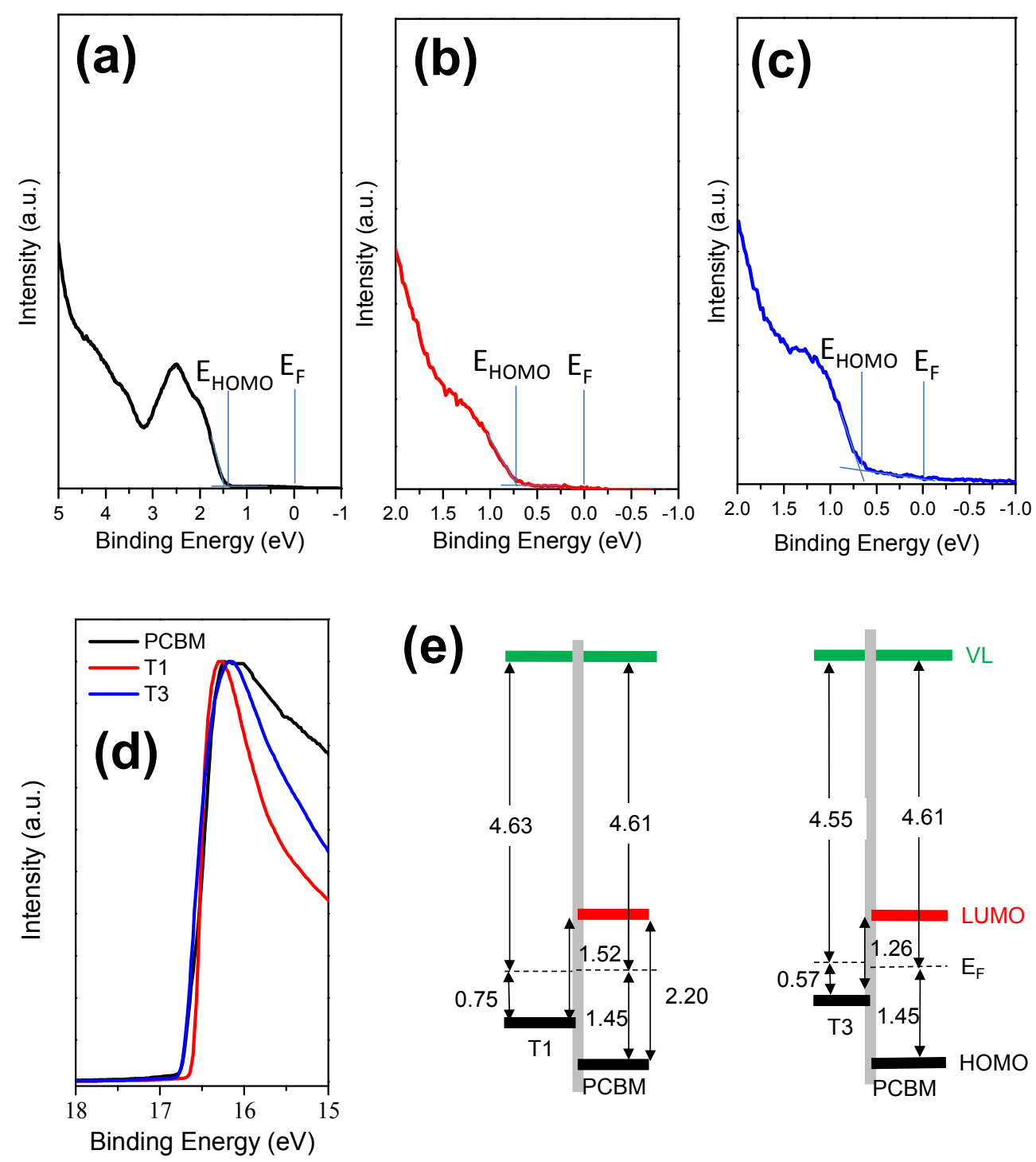

Figure S4. Photoemission spectroscopy spectra of HOMO energies of (a) $\mathrm{PC}_{71} \mathrm{BM}=1.45 \mathrm{eV}$, (b) $\mathrm{P}(\mathrm{T} 1-\mathrm{iI})=0.75 \mathrm{eV}$, and (c) $\mathrm{P}(\mathrm{T} 3-\mathrm{iI})=0.57 \mathrm{eV}$ relative to the Fermi level, $\mathrm{E}_{\mathrm{F}}$. (d) Secondary electron cutoff spectra for $\mathrm{PC}_{71} \mathrm{BM}$, T1 and $\mathrm{T} 3$, and the extracted work-functions are $4.61 \mathrm{eV}, 4.63 \mathrm{eV}$, and $4.55 \mathrm{eV}$ respectively. (e) Assuming vacuum level alignment, without interface dipole, and taking the transporting gap of $\mathrm{PC}_{71} \mathrm{BM}$ with $2.20 \mathrm{eV}$, we obtained the effective bandgap $\mathrm{E}_{\mathrm{eff}}$ of $1.58 \mathrm{eV}$ for $\mathrm{P}(\mathrm{T} 1-\mathrm{iI}): \mathrm{PC}_{71} \mathrm{BM}$ and $1.39 \mathrm{eV}$ for $\mathrm{P}(\mathrm{T} 3-\mathrm{iI}): \mathrm{PC}_{71} \mathrm{BM}$. 


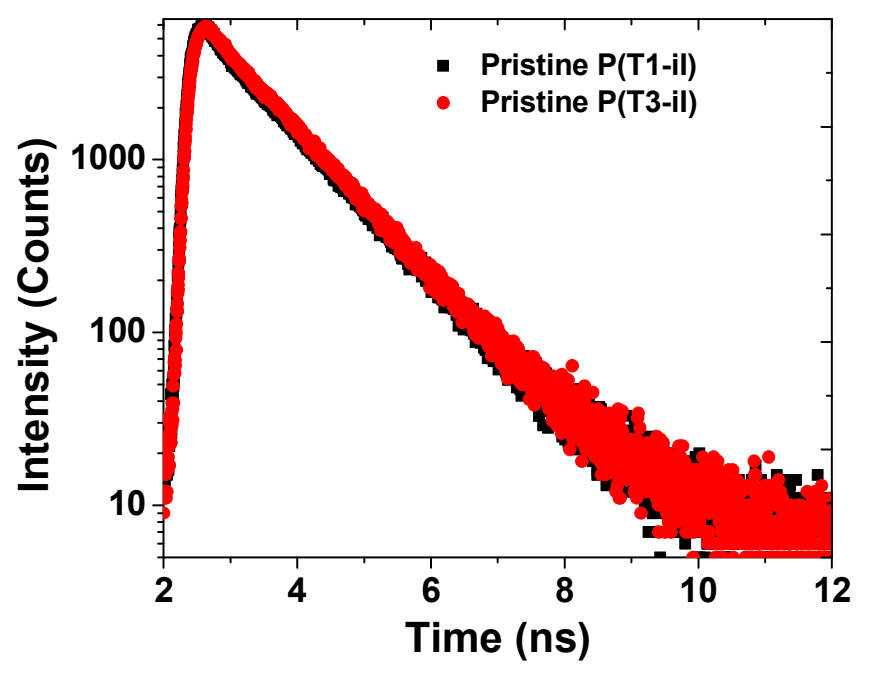

Figure S5. Transient PL for pristine P(T1-iI) and P(T3-iI) shows single exponential decays.

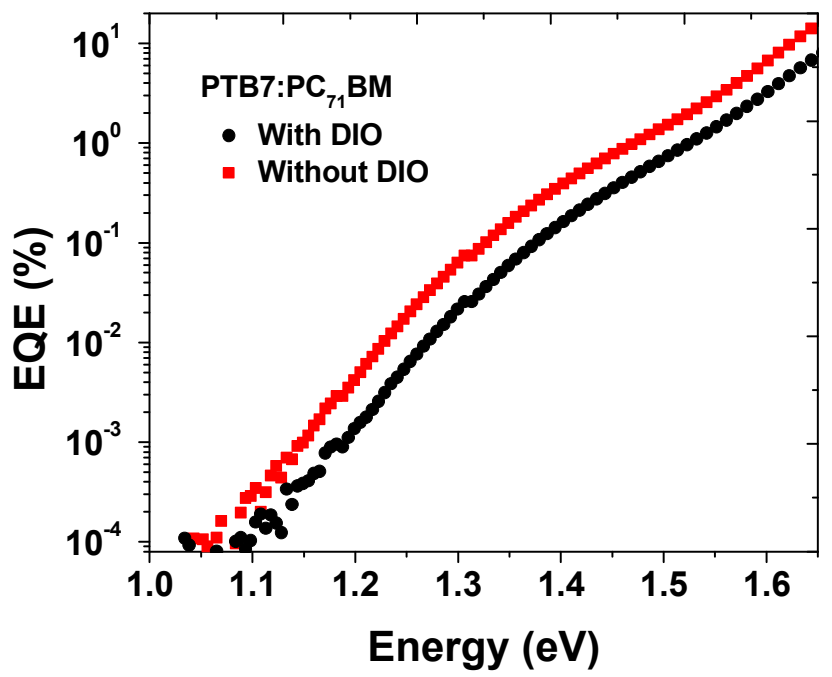

Figure S6. Sub-bandgap EQE of $\mathrm{PTB} 7: \mathrm{PC}_{71} \mathrm{BM}$ with or without $\mathrm{DIO}$ as solvent additive. No significant difference in CT cutoff is observed despite the difference in morphology.

Dielectric constant of materials used in this study:

The dielectric constants PTB7:PC 71 BM blends were measured with or without additive 1,8-Diiodooctane (DIO). The blend with DIO showed a dielectric constant of $5.03 \pm 0.22$ and the one without DIO have a dielectric constant of $4.87 \pm 0.15$. No significant difference is observed. 


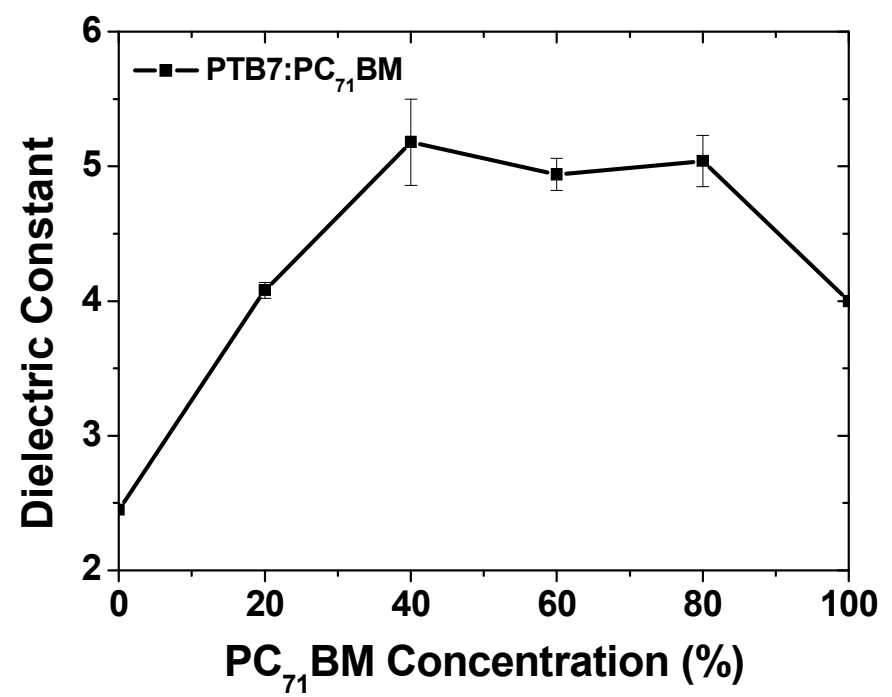

Figure S7. Dielectric constants of $\mathrm{P}(\mathrm{T} 3-\mathrm{iI}): \mathrm{PC}_{71} \mathrm{BM}$ blend film with different weight percentage of $\mathrm{PC}_{71} \mathrm{BM}$.

Density Functional Theory (DFT) Electronic Structure Calculations:

Gaussian 09 was used to perform all calculations. The alkyl groups on terthiophene and isoindigo were replaced for methyl to reduce the computational cost. The T1-iI monomer, dimer, trimer and tetramer, and T3-iI monomer, dimer, and trimer were built and optimized sequentially. The unconstrained geometries of the oligomers in gas phase were optimized by DFT using the B3LYP hybrid functional and the 6-31G (d) basis set. Frequency calculations were performed to characterize the stationary points obtained after self-consistent field convergence using the same functional/basis set method, and all minima reported here possessed no imaginary frequencies.

All Kohn-Sham orbital maps are presented as computed at the B3LYP/6-31G(d) level, and plotted using an isodensity value of $0.04 \mathrm{e} / \mathrm{bohr}^{3}$ unless otherwise noted. Kohn-Sham orbital maps are reported for T1-il model trimer and T3-iI model dimer as they exhibit a comparable number of double bonds for the two structures.

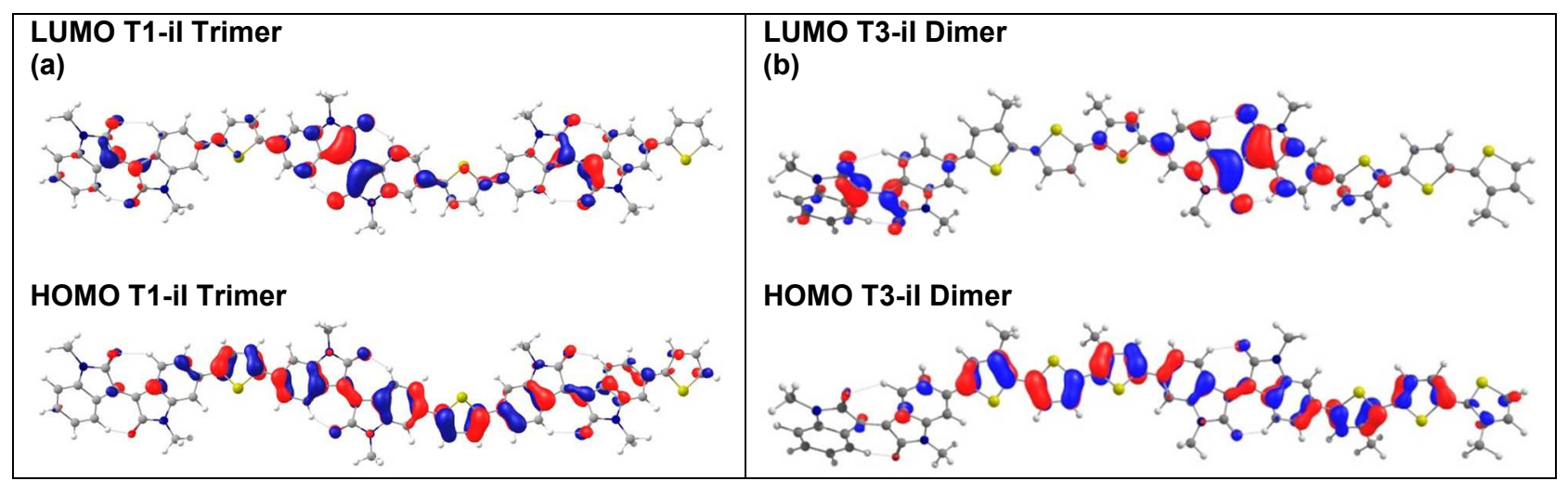

Figure S8. (a) Isodensity surfaces $\left(0.04 \mathrm{e} / \mathrm{bohr}^{3}\right)$ of the frontier orbitals and energies of a T-iI trimer. (b) Isodensity surfaces $(0.04$ $\left.\mathrm{e} / \mathrm{bohr}^{3}\right)$ of the frontier orbitals and energies of a T3-iI dimer. 